\title{
LANDSLIDE INVENTORY MAPPING FROM BITEMPORAL 10 m SENTINEL-2 IMAGES USING CHANGE DETECTION BASED MARKOV RANDOM FIELD
}

\author{
Yuanyuan Qin ${ }^{1}$, Ping $\mathrm{Lu}^{1}$,*, Zhongbin $\mathrm{Li}^{2}$ \\ ${ }^{1}$ College of Surveying and Geo-Informatics, Tongji University, 1239 Siping Road, Shanghai, China - 1731979@tongji.edu.cn, \\ luping@tongji.edu.cn \\ ${ }^{2}$ Geospatial Sciences Center of Excellence, South Dakota State University, Brookings, SD, USA - zhongbin.li@ sdstate.edu
}

\author{
Commission III, ICWG III/IVa
}

\begin{abstract}
KEY WORDS: Landslide Inventory Mapping, Change Detection, Normalized Difference Vegetation Index, Principal Component
\end{abstract} Analysis, Markov Random Field, Sentinel-2 Images

\begin{abstract}
:
Landslide inventory mapping is essential for hazard assessment and mitigation. In most previous studies, landslide mapping was achieved by visual interpretation of aerial photos and remote sensing images. However, such method is labor-intensive and timeconsuming, especially over large areas. Although a number of semi-automatic landslide mapping methods have been proposed over the past few years, limitations remain in terms of their applicability over different study areas and data, and there is large room for improvement in terms of the accuracy and automation degree. For these reasons, we developed a change detection-based Markov Random Field (CDMRF) method for landslide inventory mapping. The proposed method mainly includes two steps: 1) change detection-based multi-threshold for training samples generation and 2) MRF for landslide inventory mapping. Compared with the previous methods, the proposed method in this study has three advantages: 1) it combines multiple image difference techniques with multi-threshold method to generate reliable training samples; 2) it takes the spectral characteristics of landslides into account; and 3) it is highly automatic with little parameter tuning. The proposed method was applied for regional landslides mapping from $10 \mathrm{~m}$ Sentinel-2 images in Western China. Results corroborated the effectiveness and applicability of the proposed method especially the capability of rapid landslide mapping. Some directions for future research are offered. This study to our knowledge is the first attempt to map landslides from free and medium resolution satellite (i.e., Sentinel-2) images in China.
\end{abstract}

\section{INTRODUCTION}

In recent years, earthquake or rainfall-induced landslide hazard frequently occurred in China, leading to heavy casualties and property losses (Xu, 2012a). For landslide hazard assessment and mitigation, landslide inventory mapping recording geographic location, occurrence date, magnitude and type of landslide is required. In most previous studies, landslide inventory mapping was achieved by visual interpretation of aerial photos or remote sensing images, which however is laborintensive and time-consuming. Over the past few years, enormous methods have been proposed for landslide mapping from high resolution (HR) and even very high resolution (VHR) remote sensing images. However, limitations remain in terms of their applicability and accuracy in different study areas and data.

\subsection{Previous Work}

Previous work can be roughly divided into two parts, pixelbased and object-based.

Pixel-based methods generally take advantage of the spectral characteristic of every pixel. Lu et al. (2004a) provided a comprehensive review on change detection approaches in remote sensing community. In Van Westen and Getahun (2003a), multi-temporal landslide maps were obtained by visual interpretation of sequential aerial photos analyse evolution of the Tessina landslide in Italy. In Mondini et al. (2011a), four change variables related to landslides occurrence were selected to calibrate landslide classification models. In Hervas et al. (2003a), automatic change detection from bitemporal aerial photographs was used to map landslides in Italy. In $\mathrm{Li}$ et al. (2016a), change detection and level set method using bitemporal aerial photographs were used to map landslides in Hong Kong. In addition to change detection methods, some other methods are proposed for landslide inventory mapping. In Yang et al. (2014a), multi-temporal satellite images were used to obtain the landslide distribution map in Sichuan earthquake area on May 12, 2008. In Chen et al. (2014a), random forest was applied to detect forested landslides. However, pixel-based methods only consider spectral information and ignore the similarity among adjacent pixels, which causes a lot of salt and pepper noise in the final mapping result (Hölbling, 2017a).

Object-based methods regard adjacent pixels with similar spectral, spatial, texture information as an object. A large number of object-based landslide inventory mapping methods were developed using eCognition software. In Martha et al. (2010a), an object-oriented method using multispectral data and a digital terrain model were used to detect landslides. In Lu et al. (2011a), a semi-automatic object-oriented approach using bitemporal VHR images was applied to map the Messina landslide in Italy. In Stump et al. (2011a), object-oriented image analysis was combined with the random forest algorithm for landslide mapping. In Rau et al. (2014a), a semi-automatic object-oriented technology with multi-sensor and multispectral imagery and DEM was proposed for landslide recognition. In

\footnotetext{
* Corresponding author
} 
Pradhan et al. (2015a), an object-oriented classification method with fused data between LiDAR and VHR imagery was used to detect landslides.

Although object-based method is widely used for landslide inventory mapping, limitations still remain, such as: 1) it is difficult to find general principles to delineate different landslides because of variant features existing; 2) the labourintensive parameter tuning causes the low automation degree of landslide mapping; and 3) the accuracy of landslide mapping can be further improved.

\subsection{Our Work}

Li et al. (2016a) proposed a change detection-based Markov random field (CDMRF) for landslide inventory mapping from $0.5 \mathrm{~m}$ bitemporal aerial orthophotos over Lantau island, Hong Kong. Experimental results demonstrated that this method can map landslides accurately and efficiently. However, this method is far from perfect. We found that it has difficulty in mapping complex and low-contrast landslides from some remote sensing images. To improve the applicability and accuracy of CDMRF method over large areas, we made a further development of the method proposed in ( $\mathrm{Li}$ et al. 2016a) and proposed a new CDMRF method for landslide inventory mapping from bitemporal $10 \mathrm{~m}$ Sentinel-2 multispectral images.

\section{STUDY AREA AND DATA}

\subsection{Study Area}

The study area is located on Jiuzhaigou Country, northeast of Tibetan Qiang Autonomous Prefecture of Ngawa, Sichuan Province, China, with an area about $16.5 \mathrm{~km}^{2}$. This area features a subtropical monsoon climate with an annual average temperature of $12.7^{\circ} \mathrm{C}$ and an annual precipitation of $550 \mathrm{~mm}$. The terrain is dominated by mountains covered with dense vegetation with an annual average altitude of $4000 \mathrm{~m}$ (Liu, 2017a). An earthquake of 7.0 magnitude occurred in Jiuzhaigou Country at 21:19:46 Beijing Time on August 8, 2017. The epicenter is located at $33.20^{\circ} \mathrm{N}$ and $103.82^{\circ} \mathrm{E}$, and the depth of focus is $20 \mathrm{~km}$. The major earthquake induced a large number of shallow landslides which caused vegetation destruction and mountains exposed over seismic hazard areas. The landslides in the study area were mainly distributed along National Highway 544 from Huanglong Airport to Zhangzha Town.

\subsection{Data}

Sentinel-2 satellite is a multi-spectral Earth observation system implemented by Global Monitoring for Environment and Security (GMES) and jointly organized by the European Commission (EC) and the European Space Agency (ESA) (Drusch, 2012a). This system includes two polar-orbiting satellites (i.e., Sentinel-2A and Sentinel-2B) in the same orbit but phased at $180^{\circ}$ to each other. Together, the two satellites have a high revisit time of 5 days. The satellites carry the Multi Spectral Instrument (MSI) that samples 13 spectral bands $(10 \mathrm{~m}$, $20 \mathrm{~m}, 60 \mathrm{~m}$ bands) from visible and near-infrared (VNIR) bands to short wave infrared bands (SWIR). Level-1C and Level-2A product levels are freely available to users for land monitoring, emergency management and risk mapping (ESA, 2015).

The cloud-free pre- and post-event Sentinel-2 Level-1C multispectral images were acquired at 3:45:41 UTC on July 29, 2017 and 3:45:31 UTC on September 7, 2017, respectively and they were downloaded from the ESA Sentinel Online website
(The Copernicus Open Access Hub, 2018). Four bands, i.e., the near-infrared, red, green and blue bands with $10 \mathrm{~m}$ spatial resolution, were used in this study.

A landslide inventory map manually digitized from the pre- and post-event Sentinel-2 images was used as a reference for accuracy assessment.

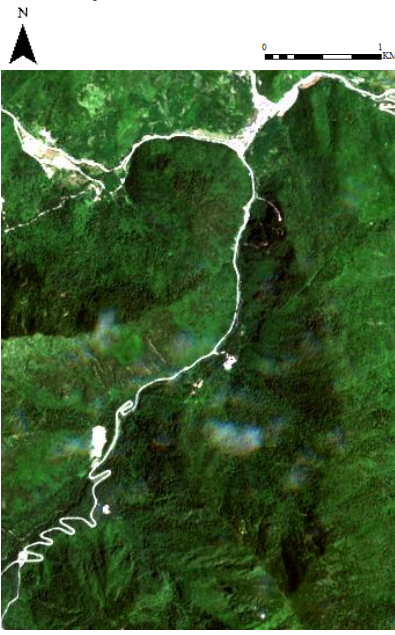

(a)

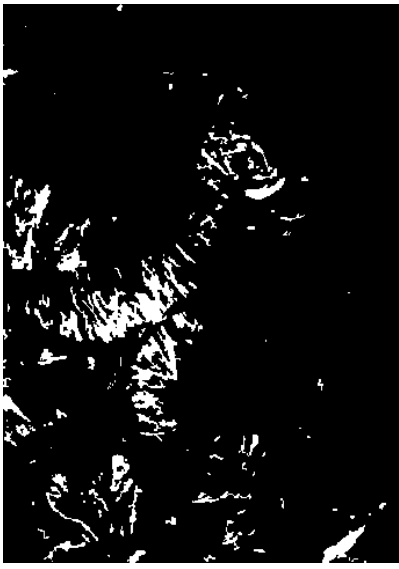

(c)

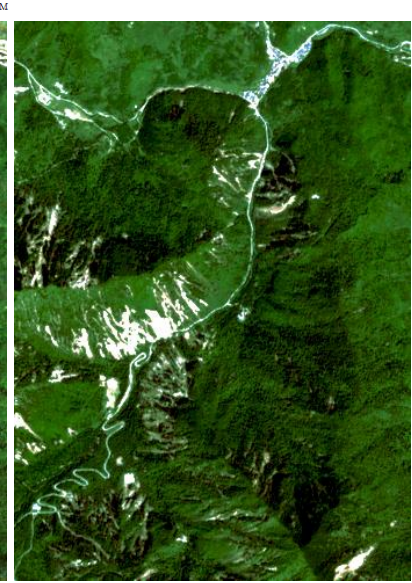

(b)

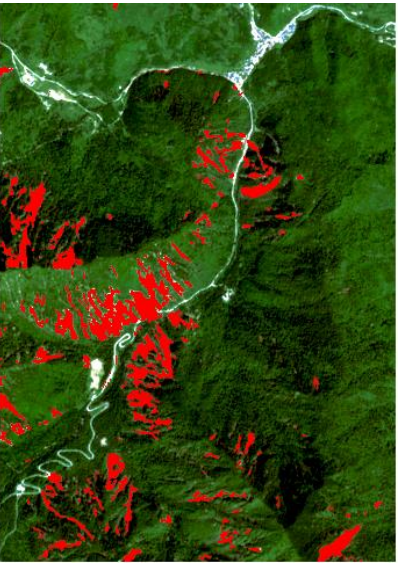

(d)
Figure 1. Test data. (a) Pre-event Sentinel-2 image. (b) Postevent Sentinel-2 image. (c) Manually digitized reference landslide inventory map. (d) Landslides in (c) overlaid on the post-event image.

\section{METHODOLOGY}

The proposed method includes four sub-steps. First, atmospheric correction was performed on Level-1C product to obtain Level-2A product, i.e., Bottom of Atmosphere (BOA) reflectance images. Then the near-infrared (NIR) and red bands of the pre- and post-event images were used to generate change detection images (CDI). Next, a multi-threshold method-based on CDI was used to generate training samples of landslides and non-landslides. Finally, the result of landslide inventory mapping is accomplished by using MRF. 


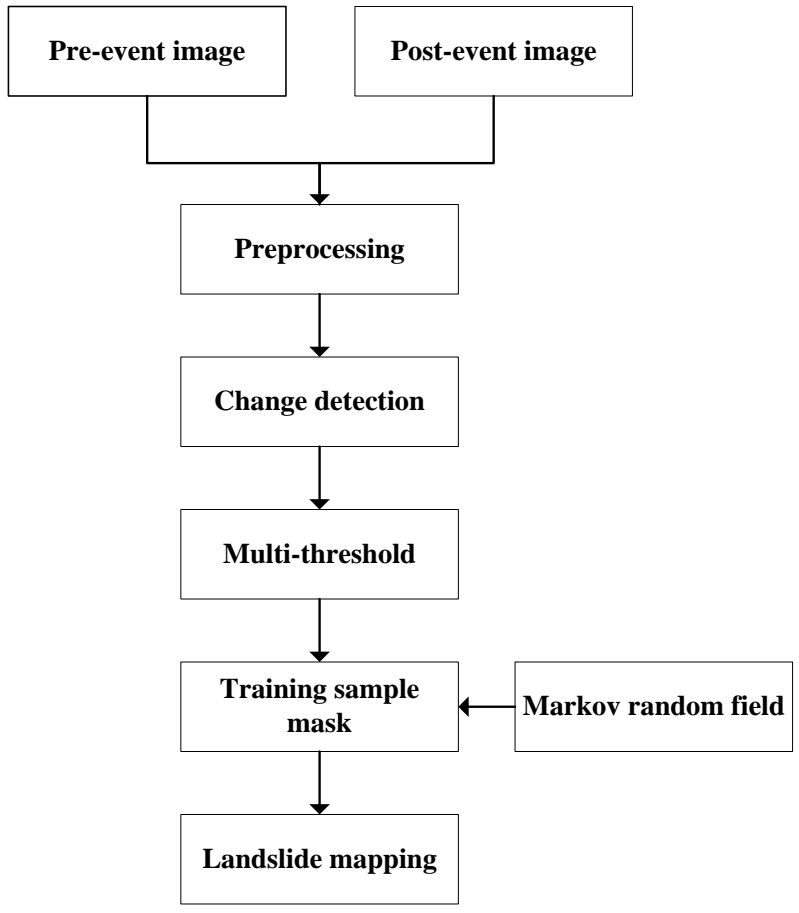

Figure 2. Flowchart of the proposed landslide mapping method

\subsection{Preprocessing of Remote Sensing Images}

Radiometric and geometric corrections have been carried out on acquired Level-1C product, including ortho-rectification and spatial registration. For the subsequent bitemporal images process and analysis, the Level-2A product was derived from the Level-1C products through the Sentinel-2 Toolbox.

\subsection{Change Detection Images}

Two image difference techniques were selected to identify shallow landslides between the pre- and post-event images.

The first image difference technique is achieved using Normalized Difference Vegetation Index (NDVI) (Mondini, 2011a). NDVI is an effective indicator of vegetation growth and cover (Lu, 2004a). The occurrence of shallow landslides leads to vegetation destruction, thus NDVI is widely used to detect landslides. In this study, changes in the pre- and post-event NDVI $\left(\delta_{\mathrm{NDVI}}\right)$ (Fig. 3(a)) were obtained by the following equation (Mondini, 2011a):

$$
\begin{aligned}
& \delta N D V I=N D V I_{\text {pre }}-N D V I_{\text {post }} \\
& =\left(\frac{N I R-r e d}{N I R+r e d}\right)_{\text {pre }}-\left(\frac{N I R-r e d}{N I R+r e d}\right)_{\text {post }}
\end{aligned}
$$

where $\quad$ NIR $=$ the near-infrared band

red $=$ red band

pre $=$ the pre-event image

post $=$ the post-event image

The second image difference technique is based on principal component analysis (PCA). PCA converts multispectral bands from multi-temporal images into linear independence components, which can reduce noise and data redundancy and increase separability between bands, and the first principal component always concentrates the most information in images

and other components account for lesser information (Lu, 2004a). Similar to NDVI, four bands, i.e., the pre- and postevent near-infrared and red bands, were used to generate four linear independence components (Mondini, 2011a). Through visual interpretation of PCA components, we found that the fourth component (PCA-4) (Fig. 3(c)) has a good match with landslides.

\subsection{Multi-Thresholding for Training Samples Generation}

$\delta_{\text {NDVI }}$ and PCA- 4 had a high correlation with the occurrence of landslides, thus they are selected to generate landslides and non-landslides training samples.

In CDI, the brighter pixels indicate larger changes which can be considered as landslides, and the darker pixels often change a little which can be considered as non-landslides. Thus, multithresholding method were applied to generate training samples of landslides and non-landslides as the following equation (Xian, 2009a; Li, 2016a):

$I_{C D I}=\left\{\begin{array}{cc}\text { landslide } & \rho(I) \geq \mu_{D I}+\left(T_{1}+T_{2}\right) * \sigma_{D I} \\ \text { uncertain area } \mu_{D I}+\left(T_{1}+T_{2}\right) * \sigma_{D I}>\rho(I)>\mu_{D I}+T_{1} * \sigma_{D I} \\ \text { non-landslide } & \rho(I) \leq \mu_{D I}+T_{1} * \sigma_{D I}\end{array}\right.$

where $\quad \rho(I)=$ brightness value of the pixel in CDI

$\mu_{D I}=$ mean value of $\mathrm{CDI}$

$\sigma_{D I}=$ standard deviation of CDI

$\mathrm{T}_{1}, \mathrm{~T}_{2}=$ parameters to set thresholds of training samples of landslides and non-landslides, and $T 1, T 2 \in R^{+}$

\begin{tabular}{|l|c|c|}
\hline CDI & \multicolumn{2}{|c|}{ Parameter } \\
\hline & $\mathrm{T}_{1}$ & $\mathrm{~T}_{2}$ \\
$\delta_{\text {NDVI }}$ & 1.8 & 1.5 \\
PCA-4 & 0.5 & 0.2 \\
\hline
\end{tabular}

Table 1. Parameters of multi-threshold method

Equation (2) explains that if the brightness value of the pixel in $\mathrm{CDI}$ is equal or greater than $\mu_{\mathrm{DI}}+\left(\mathrm{T}_{1}+\mathrm{T}_{2}\right) * \sigma \mathrm{DI}$, the pixel can be identified as the landslide sample, and if the brightness value is equal or less than $\mu_{\mathrm{DI}}+\mathrm{T} 1 * \sigma_{\mathrm{DI}}$, the pixel can be identified as the non-landslide sample. Those in this interval are classified into uncertain areas. Parameter values of $\mathrm{T}_{1}$ and $\mathrm{T}_{2}$ were set in Table 1 by trial and error.

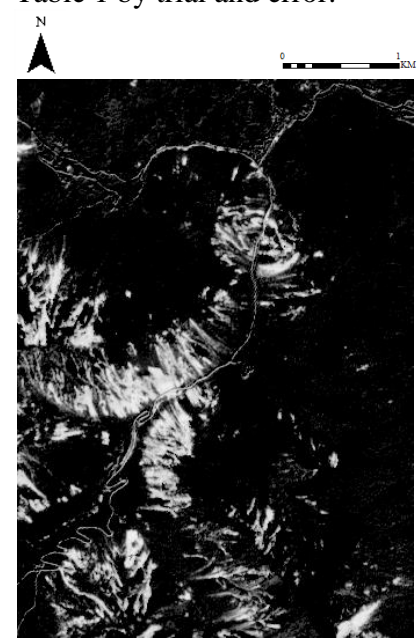

(a)

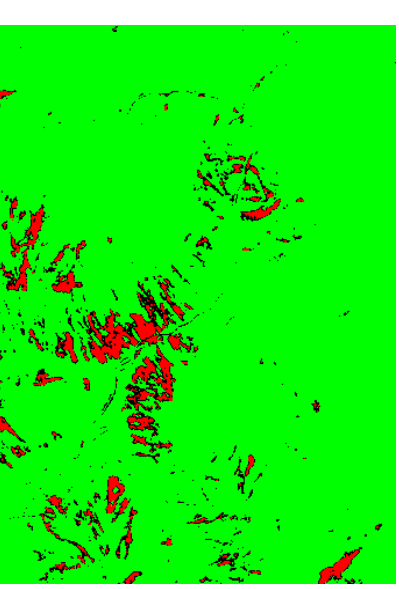

(b) 


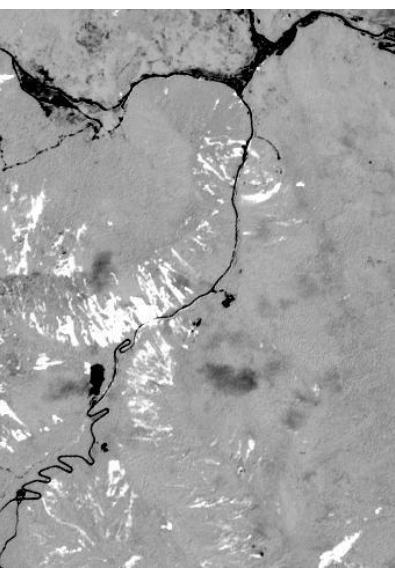

(c) (d)

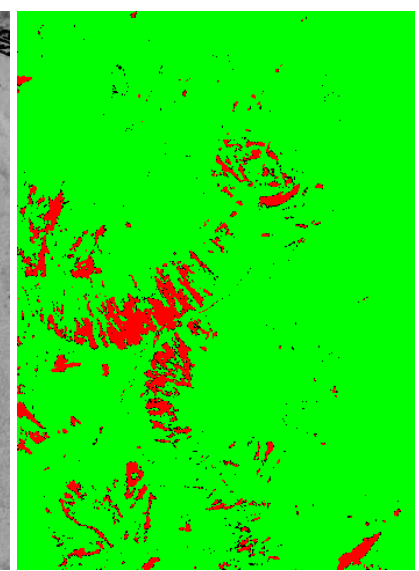

Figure 3. (a) Change detection image of bitemporal NDVI. (b) Training sample mask of $\delta_{\text {NDVI. }}$ (c) Fourth component of PCA.

(d) Training sample mask of PCA-4.

As show in Figure 3(b) and 3(d), red, green and black represent landslides, non-landslides and uncertain areas, respectively. Then, MRF method with the training sample mask and the postevent image is used to classify uncertain areas into landslide and non-landslide.

\subsection{Markov Random Field for Landslide Mapping}

First, landslide mapping is transformed into pixel labelling problem. MRF assigns a label 1 or 0 for every pixel in uncertain areas ( 1 for landslide and 0 for non-landslide), which forms a label set. And then an energy function for the label set is established using the joint probability distribution of MRF as the equation (3). Next the energy function is constructed as an undirected graph. Finally, the max-flow algorithm is used to segment the undirected graph to get the globally optimal minimum cut, which can achieve the optimal landslide mapping (Boykov, 2004a).

$$
\begin{aligned}
& E(L)=E_{u}(L)+\lambda \cdot E_{P}(L) \\
& \hat{L}=\arg \min _{L} E(L)
\end{aligned}
$$

where $\quad \mathrm{E}_{\mathrm{u}}(\mathrm{L})=$ the unary potential

$\mathrm{E}_{\mathrm{p}}(\mathrm{L})=$ the pairwise potential

$\mathrm{L}=\left(1_{1}, l_{2}, \ldots, 1_{\mathrm{n}}\right)$, a label set and $\mathrm{l}_{\mathrm{i}}$ represents the label of the ith pixel

$\lambda=\mathrm{a}$ coefficient reflecting the relative importance between the unary and pairwise potential

$\hat{L}=$ the label set correspond with the minimum of the energy function $E(L)$

3.4.1 The unary potential: $E_{u}(L)$ represents the level of similarity between the label set $\mathrm{L}$ and training samples. It is defined as the following equation (4) and (5):

$$
E_{u}(L)=\sum_{i \in C_{1}} V_{i}\left(l_{i}\right)
$$

$$
V_{i}\left(l_{i}\right)= \begin{cases}-\log \left(p\left(O \mid I_{i}\right)\right) & l_{i}=1 \\ -\log \left(p\left(B \mid I_{i}\right)\right) & l_{i}=0\end{cases}
$$

\section{where $\quad \mathrm{C}_{1}=$ the single-site clique}

$\log \left(\mathrm{p}\left(\mathrm{O} \mid \mathrm{I}_{\mathrm{i}}\right)\right)=$ the posterior probability of pixels in uncertain areas belonging to the object $\mathrm{O}$ (landslide) $\log \left(\mathrm{p}\left(\mathrm{B} \mid \mathrm{I}_{\mathrm{i}}\right)\right)=$ the posterior probability of pixels in uncertain areas belonging to the background B (nonlandslide)

$\mathrm{V}_{\mathrm{i}}\left(\mathrm{l}_{\mathrm{i}}\right)$ = negative logarithmic of the posterior probability

3.4.2 The pairwise potential: $E_{p}(L)$ represents the level of similarity between adjacent pixels (4-neighborhood). It is defined as the following equation (6), (7) and (8):

$$
\begin{aligned}
& E_{p}(L)=\sum_{(i, j) \in C_{2}} V_{i j}\left(l_{i}, l_{j}\right) \\
& V_{i j}\left(l_{i}, l_{j}\right)=\exp \left(-\beta\left(I_{i}-I_{j}\right)^{2}\right) \cdot \delta\left(l_{i}, l_{j}\right) \\
& \delta\left(l_{i}, l_{j}\right)= \begin{cases}0 & l_{i}=l_{j} \\
1 & l_{i} \neq l_{j}\end{cases}
\end{aligned}
$$

where $\quad \mathrm{C}_{2}=$ the pair-site clique

$\left(\mathrm{I}_{\mathrm{i}}-\mathrm{I}_{\mathrm{j}}\right)^{2}=$ the spectral differences of red, green and blue of visible bands between adjacent pixels

$\beta=\left(2\left\langle\left(I_{i}-I_{j}\right)^{2}\right\rangle\right)^{-1}$, and $\langle\bullet\rangle$ represents the expectation operator on the whole image

3.4.3 Energy minimization: The energy function is constructed as an undirected graph $\mathrm{G}=\langle\mathrm{V}, \mathrm{E}\rangle$. V and $\mathrm{E}$ represent the set of vertex and edge, respectively. In addition, the graph has two kinds of special vertex set, $\mathrm{S}$ and $\mathrm{T}$, which represent landslide and non-landslide training samples, respectively. In this graph, the weight of edges connecting pixels in $\mathrm{V}$ with $\mathrm{S}$ and $\mathrm{T}$ is decided by the unary potential, and the weight of edges connecting neighbouring pixels in $\mathrm{V}$ is decided by the pairwise potential. A cut in the graph $\mathrm{G}$ refers to a set of edges, which cut them can cause the set $\mathrm{V}$ divided into two disjoint sets, $V_{O}$ for landslide and $V_{B}$ for non-landslide. If the sum of weights of edges reaches a minimum, it's called the minimum cut. To achieve the optimal landslide mapping, the max-flow algorithm (Boykov, 2004a) is used to segment the undirected graph to get the globally optimal minimum cut.

\section{RESULTS AND ACCURACY ASSESSMENT}

By combining change detection based multi-threshold method with MRF, two landslide inventory mappings were obtained as shown in Figure 4. To evaluate the accuracy of the proposed method, these two mappings were compared with the manually digitized reference mapping (Fig. 1(c)). And three accuracy assessment indices: Completeness, Correctness and Quality are used as follows (Li, 2016a):

$$
\begin{aligned}
& \text { Completeness }=P_{l m} / P_{r} \\
& \text { Correctness }=P_{l m} / P_{l} \\
& \text { Quality }=P_{l m} /\left(P_{l}+P_{r u m}\right)
\end{aligned}
$$




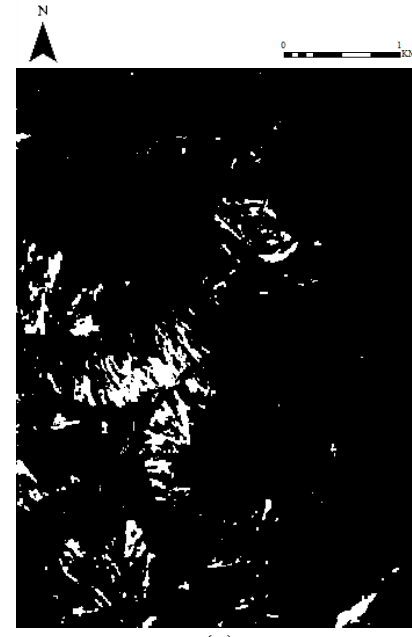

(a)

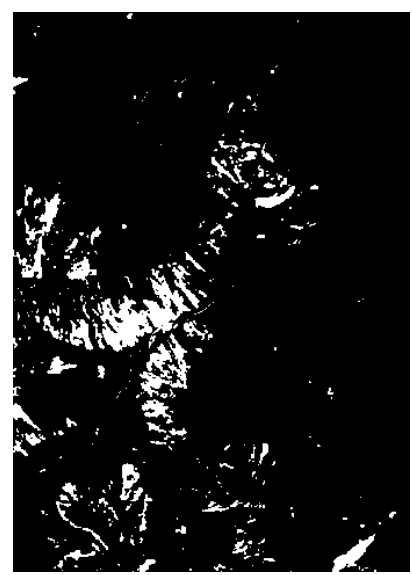

(c)

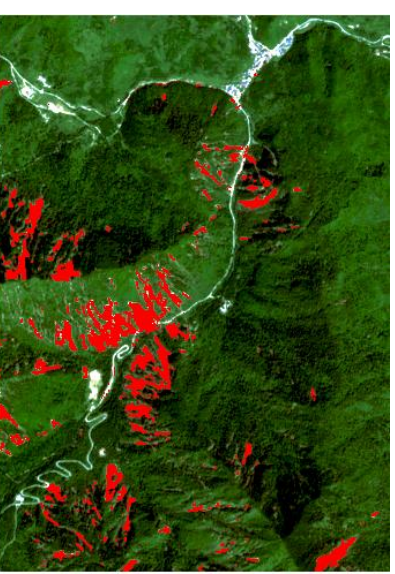

(b)

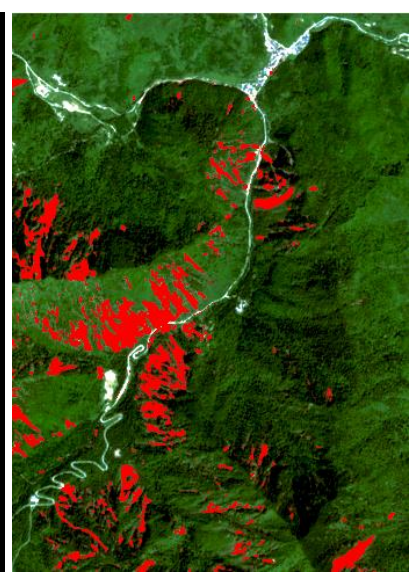

(d)

Figure 4. Landslide mapping results of MRF. (a) Binary result of using $\delta_{\text {NDVI. }}$ (b) Landslides in (a) overlaid on the post-event image. (c) Binary result of using PCA-4. (d) Landslides in (c) overlaid on the post-event image.

where $\quad \mathrm{P}_{\mathrm{Im}}=$ the total pixel number of the mapped landslides that are consistent with the truths

$\mathrm{P}_{\mathrm{r}}=$ the total pixel number of the truths

$\mathrm{P}_{1}=$ the total pixel number of the mapped landslides $\mathrm{P}_{\text {rum }}=$ the total pixel number of the truths are inconsistent with the mapped landslides

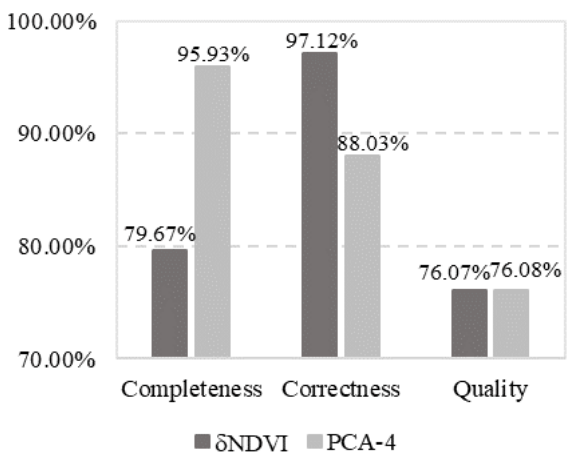

Figure 5. The histogram of accuracy assessment for LIM in the study area
It has been calculated that in the study area, using bitemporal NDVI ( $\left.\delta_{\mathrm{NDVI}}\right)$ for CDI and MRF for mapping landslides, the completeness, correctness, and quality of LIM achieved $79.67 \%$, $97.12 \%$ and $76.07 \%$, respectively. Using PCA-4 instead of $\delta_{\mathrm{NDVI}}$, the completeness of $95.93 \%$, the correctness of $88.03 \%$, and the quality of $76.08 \%$ were achieved. It shows clearly that in the study area, using $\delta_{\mathrm{NDVI}}$ for CDI can achieve more correct mapping result but the omission error is more than PCA-4. And the PCA-4 would cause excessive landslide mapping. The quality of both methods is almost the same, which indicates the proposed new CDMRF can achieve mapping landslide inventory rapidly and accurately over large areas.

\section{CONCLUSION}

In this study, we integrated two image difference technologies into the CDMRF for automatic and accurate landslide inventory mapping from bitemporal $10 \mathrm{~m}$ Sentinel-2 multispectral images. First, a difference image based on bitemporal NDVI was generated and the fourth band image of principal component analysis (PCA-4) was generated using the pre- and post-event near-infrared (NIR) and red bands. Second, multi-threshold method based on generated change detection images (CDI) was used to generate training sample masks, which include landslides, non-landslides and uncertain pixels. Finally, using Markov random field (MRF) with training sample masks onto the post-event Sentinel-2 image achieved the optimal landslide inventory mapping (LIM).

The proposed method was used to map landslides in an area about $16.5 \mathrm{~km}^{2}$ in Western China. $\delta_{\text {NDVI }}$ for CDI achieved completeness and correctness of $79.67 \%$ and $97.12 \%$ and PCA4 for CDI achieved completeness and correctness of $95.93 \%$ and $88.03 \%$. The quality of these two methods achieved $76.07 \%$ and $76.08 \%$, respectively.

Experimental results verified the effectiveness of the proposed method: 1) it takes advantage of spectral characteristics of landslides; 2) it takes into account the spatial contextual information in post-event remote sensing image, which makes up for the deficiency of ignoring spatial characteristic of pixels in traditional pixel-based methods; 3) it has a high degree of automation due to little parameter tuning; and 4) it is a generic landslide inventory mapping method. This method has great potential to provide a technical support for future landslide inventory mapping over large areas in China.

\section{REFERENCES}

Boykov, Y., and Jolly, M. P., 2001a, Interactive Graph Cuts for Optimal Boundary \& Region Segmentation of Objects in N-D Images. IEEE International Conference on Computer Vision IEEE Computer Society, pp.105.

Boykov, Y., and Kolmogorov, V., 2004a. An experimental comparison of min-cut/max-flow algorithms for energy minimization in vision. IEEE Transactions on Pattern Analysis \& Machine Intelligence, 26(9), pp. 1124-1137.

Chen, W., Li, X., Wang, Y., Chen, G., and Liu, S., 2014a. Forested landslide detection using lidar data and the random forest algorithm: a case study of the three gorges, china. Remote Sensing of Environment, 152, pp. 291-301. 
Drusch, M., Bello, U. D., Carlier, S., Colin, O., Fernandez, V., and Gascon, F., et al., 2012a. Sentinel-2: esa's optical highresolution mission for gmes operational services. Remote Sensing of Environment, 120(1), pp. 25-36.

ESA, 2015, Sentinel-2 User Handbook.

Hervás, J., Barredo, J. I., Rosin, P. L., Pasuto, A., Mantovani, F., and Silvano, S., 2003a. Monitoring landslides from optical remotely sensed imagery: the case history of tessina landslide, italy. Geomorphology, 54(1-2), pp. 63-75.

Hölbling, D., Eisank, C., Albrecht, F., Vecchiotti, F., Friedl, B., and Weinke, E., et al., 2017a. Comparing manual and semiautomated landslide mapping based on optical satellite images from different sensors. Geosciences, 7(2), pp. 37.

Li, Z., Shi, W., Lu, P., Yan, L., Wang, Q., and Miao, Z., 2016a. Landslide mapping from aerial photographs using change detection-based markov random field. Remote Sensing of Environment, 187, pp. 76-90.

Li, Z., Shi, W., Myint, S. W., Lu, P., and Wang, Q., 2016a. Semi-automated landslide inventory mapping from bitemporal aerial photographs using change detection and level set method. Remote Sensing of Environment, 175, pp. 215-230.

Liu, J., Wang, T., Shi, J., and Li, Z., 2017a. Emergency rapid assessment of landslides induced the Jiuzhaigou Ms 7.0 earthquake, Sichuan, China. Journal of Geomechanics, 23(5), pp. 639-645.

Lu, D., Mausel, P., Brondizio, E., and Moran, E., 2004a. Change detection techniques. int $\mathrm{j}$ remote sens. International Journal of Remote Sensing, 25(12), pp. 2365-2401.

Lu, P., Stumpf, A., Kerle, N., and Casagli, N., 2011a. Objectoriented change detection for landslide rapid mapping. IEEE Geoscience \& Remote Sensing Letters, 8(4), pp. 701-705.

Martha, T. R., Kerle, N., Jetten, V., Westen, C. J. V., and Kumar, K. V., 2010a. Characterising spectral, spatial and morphometric properties of landslides for semi-automatic detection using object-oriented methods. Geomorphology, 116(1), pp. 24-36.

Mondini, A. C., Guzzetti, F., Reichenbach, P., Rossi, M., Cardinali, M., and Ardizzone, F., 2011a. Semi-automatic recognition and mapping of rainfall induced shallow landslides using optical satellite images. Remote Sensing of Environment, 115(7), pp. 1743-1757.

Pradhan, B., Jebur, M. N., Shafri, H. Z. M., and Tehrany, M. S., 2016a. Data fusion technique using wavelet transform and taguchi methods for automatic landslide detection from airborne laser scanning data and quickbird satellite imagery. IEEE Transactions on Geoscience \& Remote Sensing, 54(3), pp. $1610-1622$.

Rau, J. Y., Jhan, J. P., and Rau, R. J., 2013a. Semiautomatic object-oriented landslide recognition scheme from multisensor optical imagery and dem. IEEE Transactions on Geoscience \& Remote Sensing, 52(2), pp. 1336-1349.
Rother, C., Kolmogorov, V., and Blake, A., 2004a. "grabcut": interactive foreground extraction using iterated graph cuts. Trans. on Graphics, 23(3), pp. 309-314.

Stumpf, A., and Kerle, N., 2011a. Object-oriented mapping of landslides using random forests. Remote Sensing of Environment, 115(10), pp. 2564-2577.

The Copernicus Open Access Hub, 2018. Version 0.14.1-3, https://scihub.copernicus.eu/dhus/\#/home

Ventura, G., Vilardo, G., Terranova, C., and Sessa, E. B., 2011a. Tracking and evolution of complex active landslides by multitemporal airborne lidar data: the montaguto landslide (southern italy). Remote Sensing of Environment, 115(12), pp. 3237-3248.

Xian, G., Homer, C., and Fry, J., 2009a. Updating the 2001 national land cover database land cover classification to 2006 by using landsat imagery change detection methods. Remote Sensing of Environment, 113(6), pp.1133-1147.

Xu, C., Xu, X., Dai, F., and Saraf, A. K., 2012a. Comparison of different models for susceptibility mapping of earthquake triggered landslides related with the 2008 wenchuan earthquake in china. Computers \& Geosciences, 46(3), pp. 317-329.

Yang, X. J., and Chen, L. D., 2010a. Using multi-temporal remote sensor imagery to detect earthquake-triggered landslides. International Journal of Applied Earth Observation \& Geoinformation, 12(6), pp. 487-495. 